\title{
NECESSARY CONDITIONS FOR A MATHEMATICAL PROGRAMMING PROBLEM WITH SET AND CONE CONSTRAINTS
}

\author{
YOUJI NAGAHISA ${ }^{1}$
}

(Received March 27, 1986; revised February 18, 1987)

\begin{abstract}
This paper is devoted to the derivation of a necessary condition of F. John type which must be satisfied by a solution of a mathematical programming problem with set and cone constraints. The necessary condition is applied to an optimisation problem defined on functional spaces with inequality state constraints. Furthermore a pseudo open mapping theorem is developed in the course of proving the main theorem.
\end{abstract}

\section{Introduction and notations}

Optimality conditions for the solution of the following mathematical programming problem in infinite-dimensional spaces have been studied by Varaiya [8], Borwein [2], Kurcyusz [6], Zowe and Kurcyusz [9] and others:

$$
\operatorname{minimise}\{f(x) ; x \in X, w(x) \in W\} \text {. }
$$

Many of them assume that the cone $W$ has an interior. This excludes the canonical non-positive cone of an $L^{p}$-space, as was pointed out by Craven [3]. However, many cones of the constraints can be represented as a product of two cones such that one has an interior and the other does not. For example, when we consider the programming problem on function spaces, the former represents the terminal constraints and the latter the state constraints. Hence we shall concern ourselves in this paper with a necessary condition of F. John type for the following mathematical programming problem:

$$
\text { minimise }\{f(x) ; x \in X, g(x) \in Y, h(x) \in Z\} \text {. }
$$

1 Department of Mathematics, Faculty of Education, Yamaguchi University, Yosida, Yamaguchi, 753, Japan

(C) Copyright Australian Mathematical Society 1988, Serial-fee code 0334-2700/88 
Here $f$ is a real-valued function on a real normed space $\mathfrak{X} ; g$ and $h$ are mappings from $\mathfrak{X}$ into real normed spaces $\mathfrak{Y}$ and $\mathcal{Z}$, respectively; $X$ is a non-empty subset of $\mathfrak{X} ; Y$ is a convex cone in $\mathfrak{Y}$ with non-empty interior and $Z$ is a convex cone in 3. Throughout this paper, a set $C$ of the underlying normed space will be called a cone, if it is not empty and if $\mu C \subset C$ whenever $\mu>0$.

As regards notation and terminology, $\mathfrak{Y}^{*}$ and $\mathfrak{Z}^{*}$ shall denote the conjugate spaces of $\mathfrak{Y}$ and $\mathcal{Z}$, respectively. $Y^{*}$ and $Z^{*}$ shall respectively denote the polar cones of the cones $Y$ and $Z$, that is

$$
\begin{aligned}
& Y^{*}=\left\{y^{*} \in \mathfrak{Y}^{*} ; y^{*}(y) \leq 0 \text { for any } y \in Y\right\}, \\
& Z^{*}=\left\{z^{*} \in \mathfrak{Z}^{*} ; z^{*}(z) \leq 0 \text { for any } y \in Z\right\} .
\end{aligned}
$$

For a subset $A$ and an element $a$ of the underlying normed space, int. $A$, cl. $A$ and $[A \mid a]$ shall denote respectively the interior of $A$, the closure of $A$ and the conical hull $A-a$, that is

$$
[A \mid a]=\bigcup_{\lambda>0}\{\lambda(A-a)\} .
$$

By convention, each origin of the spaces $\mathfrak{X}, \mathfrak{Y}$ and $\mathfrak{Z}$ shall be denoted by the same notation $\theta$ and each origin of the conjugate spaces $\mathfrak{Y}^{*}$ and $\mathfrak{Z}^{*}$ shall be denoted by the same notation $\theta^{*}$. Also the same notation $\|\cdot\|$ shall denote each norm of the spaces $\mathfrak{X}, \mathfrak{Y}$ and $\mathfrak{Z}$, unless explicitly stated otherwise. An element $x$ shall be called a feasible solution of (P) if $x \in M=X \cap g^{-1}(Y) \cap h^{-1}(Z)$. An element $\hat{x} \in M$ shall be called an optimal solution of $(\mathrm{P})$ if $\hat{x}$ satisfies

$$
f(x)=\min _{x \in M} f(x) .
$$

Usually constraint qualifications are imposed on the problem (P) in order to ensure that Lagrange multipliers of Kuhn-Tucker type, rather than only of F. John type, exist. Even in that case, certain qualifications must be imposed on the problem (P) for the lack of the hypothesis that int. $Z \neq 0$ (see e.g., $[1,3]$ ). Now we will suppose throughout this paper that the following is satisfied by the spaces $\mathfrak{X}, \mathfrak{Z}$ and the cone $Z$ in order to ensure that Lagrange multipliers of $F$. John type exist.

AsSUMPTION. Besides the original norms denoted by $\|\cdot\|$, we introduce additional norms on the linear spaces $\mathfrak{X}$ and $\mathfrak{Z}$, which are denoted by the same notation $|\cdot|$, with the following properties:

(i) the cone $Z$ has non-empty interior with respect to the additional norm $|\cdot|$,

(ii) there exists an $\alpha>0$ such that

$$
\|x\| \leq \alpha|x|, \quad\|z\| \leq \alpha|z| \quad \text { for all } x \in \mathfrak{X} \text { and } z \in \mathfrak{Z} .
$$

For a subset $A$ of $\mathfrak{X}$ (or 3 respectively), $\bar{A}$ and $A^{0}$ denote the closure and the interior of $A$ with respect to the additional norm $|\cdot|$. Furthermore, for an 
element $\tilde{x} \in X, T(X, \tilde{x})$ denotes a sequential cone to $X$ at $\tilde{x}$ (cf. $[8,9])$, that is

$$
\begin{aligned}
T(X, \tilde{x})=\left\{x \in X ; \lim _{n \rightarrow \infty} \lambda_{n}=0, \lim _{n \rightarrow \infty}\left|x-\left(x_{n}-\tilde{x}\right) / \lambda_{n}\right|=0,\right. \\
\left.x_{n} \in X, \lambda_{n}>0 \text { for } n=1,2, \ldots\right\} .
\end{aligned}
$$

DEFinition. An element $\hat{x} \in \mathfrak{X}$ is called a non-singular point of $(P)$, if

(i) there exist continuous linear operators $F_{\hat{x}}: \mathfrak{X} \rightarrow R, G_{\hat{x}}: \mathfrak{X} \rightarrow \mathfrak{Y}$ and a linear operator $H_{\hat{x}}: \mathfrak{X} \rightarrow \mathfrak{Y}$ such that for each $x \in \mathfrak{X}$,

$$
\begin{aligned}
& \lim _{\substack{\lambda \downarrow 0 \\
|u-x| \rightarrow 0}}\left|\frac{f(\hat{x}+\lambda u)-f(\hat{x})}{\lambda}-F_{\hat{x}}(x)\right|=0, \\
& \lim _{\substack{\lambda \neq 0 \\
|u-x| \rightarrow 0}}\left|\frac{g(\hat{x}+\lambda u)-g(\hat{x})}{\lambda}-G_{\hat{x}}(x)\right|=0, \\
& \lim _{\substack{\lambda \downarrow 0 \\
|u-x| \rightarrow 0}}\left|\frac{h(\hat{x}+\lambda u)-h(\hat{x})}{\lambda}-H_{\hat{x}}(x)\right|=0,
\end{aligned}
$$

(ii) there exists a convex subset $Q$ of $\mathfrak{X}$ such that $\theta \in Q \subset T(X, \hat{x})$,

(iii) we can find a subset $K$ of 3 and a positive $\beta$ such that $\bar{K}=\mathfrak{Z}$ and for each $z \in K$, there exists an $x \in \overline{[Q \mid \theta]}$ satisfying $z \in H_{\hat{x}}(x)-\overline{[Z \mid h(\hat{x})]}$ and $\|x\| \leq \beta\|z\|$.

The main purpose of this paper is to prove the following necessary condition of $F$. John type for an optimal solution of $(P)$.

THEOREM 1. If the space $\mathfrak{X}$, the $\mathfrak{Z}$ and the cone $Z$ satisfy the Assumption and if an optimal solution $\hat{x}$ of $(\mathrm{P})$ is non-singular, then there exist Lagrange multipliers $\eta \geq 0, y^{*} \in Y^{*}$ and $z^{*} \in Z^{*}$ such that

$$
\begin{gathered}
\left(\eta, y^{*}\right) \neq\left(0, \theta^{*}\right), \\
y^{*}(g(\hat{x}))=0, \quad z^{*}(h(\hat{x}))=0 \quad \text { and } \\
\eta \cdot F_{\hat{x}}(x)+y^{*}\left(G_{\hat{x}}(x)\right)+z^{*}\left(H_{\hat{x}}(x)\right) \geq 0 \quad \text { for all } x \in Q,
\end{gathered}
$$

where $F_{\hat{x}}, G_{\hat{x}}, H_{\hat{x}}$ and $Q$ are as indicated in the Definition.

There have been many works in the area considered in this paper. In [9], Zowe and Kurcyusz have obtained necessary conditions of Kuhn-Tucker type for the problem $(\tilde{\mathrm{P}})$, where the cone $W$ does not have an interior, assuming closed convexity of the set $X$, completeness of the spaces and regularity. On applying their results to practical problems, it frequently happens that the underlying spaces must be completed. In addition, the regularity condition must be satisfied in this completion. But the task is difficult and this kind of nice completion sometimes fails to be realised. 
Since the cone $Z$ has a non-empty interior with respect to the additional norm $|\cdot|$, we can show, using the result of [7], the existence of Lagrange multipliers like those of Theorem 1 even with the exclusion of (ii) from the Assumption and (iii) from the Definition. While $z^{*}$ in the multiplier is an element of the space $\mathcal{Z}$ of all linear functionals continuous with respect to the additional norm $|\cdot|$, it is not guaranteed, in this case, that $z^{*} \in 3^{*}$. Borwein has obtained necessary conditions of F. John type using two different kinds of topologies (Theorem 3 as well as its Remark in [2]). But his results do not give an assurance that $z^{*} \in 3^{*}$, because $z^{*}$ must be continuous with respect to the topology that the cone $Z$ has a non-empty interior.

In practice many examples exist for function spaces conforming to our Assumption. In these cases, the space $\mathfrak{Z}^{*}$ is a proper linear subset of the space $\mathfrak{Z}^{\prime}$. Hence the space $\mathfrak{Z}^{*}$ is different in quality to the space $\mathbf{3}^{\prime}$. Then it is important to ensure that $z^{*} \in 3^{*}$. The aim of this paper is to give this assurance, that is, that the linear functional $z^{*}$ is continuous with respect to the original norm $\|\cdot\|$. It is (i) of the Assumption and (iii) of the Definition that give this assurance.

In Section 2 we show some mathematical concepts and pseudo-open mapping properties. In Section 3 we give the proof of Theorem 1. Finally, in Section 4 we apply Theorem 1 to an example and give a concluding remark.

\section{Preliminary results and pseudo open mapping property}

In this section, we present, under the Assumption of Section 1, a pseudo-open mapping property which will be used in the sequel.

We know the following properties about a convex set of a normed space (see Lemma 11.A in [5]).

LEMMA 1. Let $A$ and $B$ be convex subset of 3 with non-empty interior with respect to the original norm $\|\cdot\|$ and the additional norm $|\cdot|$, respectively. Then

$$
\begin{array}{ll}
\text { cl. } A=\text { cl. }\{\text { int. } A\}, & \text { int. } A=\text { int. }\{\text { cl. } A\}, \\
\bar{B}=\overline{\left\{B^{0}\right\}}, & B^{0}=\{\bar{B}\}^{0} .
\end{array}
$$

Using the properties of a convex cone in a normed space, we can easily show the following lemma. Therefore we omit the proof.

LEMMA 2. If $A$ and $B$ are convex cones in 3 and if $z$ is an element of 3 , then

(i) cl. $A$ and $\bar{A}$ are both convex cones in 3 . If in addition, $A$ and $B$ have nonempty interiors with respect to the original norm $\|\cdot\|$ and the additional 
norm $|\cdot|$ respectively, then

(ii) int. $A$ and $B^{0}$ are both convex cones in $\mathcal{Z}$,

$$
A+\operatorname{int} . A=\text { cl. } A+\text { int. } A=\text { int. } A \text {, }
$$

$$
B+B^{0}=\bar{B}+B^{0}=B^{0},
$$

(iv) $[A \mid z]$ and $[B \mid z]$ are both convex cones in 3 with int. $A \subset[A \mid z]$ and $B^{0} \subset$ $[\bar{B} \mid z]$,

(v) int. $[A \mid z]=[$ int. $A \mid z]$ and $[B \mid z]^{0}=\left[B^{0} \mid z\right]$.

Using the Assumption, one can show that for every $A$ of $\mathcal{Z}, \bar{A} \subset \operatorname{cl} . A$. By virtue of Lemma 1 , we have for every convex subset $A$ of 3 with $A^{0} \neq \varnothing$, $\bar{A} \subset \overline{\left\{A^{0}\right\}} \subset \operatorname{cl} .\left\{A^{0}\right\}$. Hence it follows, from Assumption and (iv) of Lemma 2, that

$$
\overline{[Z \mid \hat{z}]} \subset \operatorname{cl} .\left\{[Z \mid \hat{z}]^{0}\right\} \quad \text { for every } \hat{z} \in \mathcal{Z} .
$$

We now present the following result, which may be not inaptly termed the pseudo-open mapping property (cf. Section 2 in [9]).

THEOREM 2. Let $C$ be a convex cone in $\mathfrak{X}$ and let $L$ be a linear operator from $\mathfrak{X}$ into $\mathfrak{Z}$. Suppose (i) the spaces $\mathfrak{X}, \mathfrak{Z}$ and the cone $Z$ satisfy the Assumption, (ii) we can find a positive $\beta$ and a subset $K$ of 3 such that $\bar{K}=3$ and, for each $z \in K$, there exists an $x \in C$ satisfying

$$
z \in L(x)-\bar{Z} \text { and }\|x\| \leq \beta\|z\| .
$$

Then, for each open subset $O$ of $\mathfrak{X}$ (with respect to the original norm $\|\cdot\|$ ), the set $L(C \cap O)-Z^{0} \subset \mathcal{Z}$ is open (with respect to the original norm $\|\cdot\|$ ).

ProOF. For $\rho>0$, let $U_{\rho}$ and $V_{\rho}$ be respectively defined by

$$
U_{\rho}=\{x \in \mathfrak{X} ;\|x\|<\rho\} \quad \text { and } \quad V_{\rho}=\{z \in \mathfrak{Z} ;\|z\|<\rho\} .
$$

We first show the following statement:

(S) for any $\varepsilon>0$, there exists a $\delta>0$ such that

$$
V_{\delta} \subset L\left(C \cap U_{\varepsilon}\right)-\bar{Z} \text {. }
$$

Let $\varepsilon$ be an arbitrary positive number. We set $\delta=\varepsilon / \beta(>0)$. By virtue of (ii) of the Assumption, it is easily shown that

$$
\left\{V_{\delta}\right\}^{0}=V_{\delta}=\overline{\left\{K \cap V_{\delta}\right\}},
$$

because $\bar{K}=3$. By our hypotheses, it is implied that $\left\{K \cap V_{\delta}\right\} \subset L\left(C \cap U_{\varepsilon}\right)-\bar{Z}$, which, by virtue of $(7)$, implies that

$$
\left\{V_{\delta}\right\}^{0}=V_{\delta} \subset \overline{\left\{L\left(C \cap U_{\varepsilon}\right)-\bar{Z}\right\}} .
$$


It follows from (i) of the Assumption that $L\left(C \cap U_{\varepsilon}\right)-\bar{Z}$ is a convex subset of $\mathfrak{Z}$ with $\left\{L\left(C \cap U_{\varepsilon}\right)-\bar{Z}\right\}^{0}=\varnothing$. Hence, by virtue of Lemma 1 , we obtain that

$$
\left.\left\{\overline{\left\{L\left(C \cap U_{\varepsilon}\right)-\bar{Z}\right.}\right\}\right\}^{0}=\left\{L\left(C \cap U_{\varepsilon}\right)-\bar{Z}\right\}^{0} \subset L\left(C \cap U_{\varepsilon}\right)-\bar{Z} .
$$

This together with (8) imply that $V_{\delta} \subset L\left(C \cap U_{\varepsilon}\right)-\bar{Z}$, thus proving the statement (S).

We now turn to the proof of the theorem. Let $O$ be an arbitrary open subset of $\mathfrak{X}$. If $C \cap O=\varnothing$, then $L(C \cap O)-Z^{0}$ will be considered to be the empty set, which is open. Hence we assume that $C \cap O \neq \varnothing$. Let $z \in L(C \cap O)-Z^{0}$ be arbitrary; then there is an $x \in C \cap O$ such that $z \in L(x)-Z^{0}$. Since $O$ is open, there is an $\varepsilon>0$ such that $x+U_{\varepsilon} \subset O$. It follows from (S) that there is a $\delta>0$ such that $V_{\delta} \subset L\left(C \cap U_{\varepsilon}\right)-\bar{Z}$. The linearity of $L$ together with (iii) of Lemma 2 implies that

$$
\begin{aligned}
z+V_{\delta} & \subset L(x)+L\left(C \cap U_{\varepsilon}\right)=\left(\bar{Z}+Z^{0}\right) \\
& \subset L\left(C \cap\left\{x+U_{\varepsilon}\right\}\right)-Z^{0} \subset L(C \cap O)-Z^{0} .
\end{aligned}
$$

That is, $z$ is an interior point of $L(C \cap O)-Z^{0}$. Consequently the set $L(C \cap O)-Z^{0}$ is open, completing the proof of the theorem.

\section{Proof of Theorem 1}

This section will be devoted to the proof of Theorem 1 under the Assumption. Throughout this section let $\hat{x}$ be an optimal solution of $(\mathrm{P})$, as well as a nonsingular element. Let the mappings $F_{\hat{x}}, G_{\hat{x}}, H_{\hat{x}}$, the sets $Q, K$ and the positive number $\beta$ be as indicated in the Definition. We denote by $O$ the subset of $\mathfrak{X}$ defined by

$$
O=\left\{x \in \mathfrak{X} ; F_{\hat{x}}(x)<0, G_{\hat{x}}(x) \in \operatorname{int} .[Y \mid g(\hat{x})]\right\} .
$$

We begin with the following lemma.

LEMMA 3. If

$$
H_{\hat{x}}(\overline{|Q| \theta]} \cap O) \cap[Z \mid h(\hat{x})]^{0}=\varnothing,
$$

then there exist $\eta \in R, y^{*} \in Y^{*}$ and $z^{*} \in Z^{*}$ which satisfy (3)-(5).

Proof. Since $Q$ is a convex set with $\theta \in Q$, it follows, from (i) of Lemma 2 and (1), that $\overline{[Q \mid \theta]}$ is a convex cone in $\mathfrak{X}$ such that $\theta \in \overline{[Q \mid \theta]}$. Furthermore, by virtue of (i) of the Assumption and (iv) of Lemma $2,[Z \mid h(\hat{x})]$ is a convex cone in 3 such that $\overline{[Z \mid h(\hat{x})]}$.

We first consider the case where $\overline{[Q \mid \theta]} \cap O \neq \varnothing$. By (i) of the Definition, the subset $O$ of $\mathfrak{X}$ is a convex cone which is open (with respect to the original norm $\|\cdot\|)$. Since $\hat{x}$ is a non-singular point of $(P)$, it follows from (9) and Theorem 2 that the set $H_{\hat{x}}(\overline{[Q \mid \theta]} \cap O)-[Z \mid h(\hat{x})]^{0}$ does not contain the origin $\theta$ of $\mathfrak{Z}$, and 
that it is an open convex cone in $\mathfrak{Z}$ (with respect to the original norm $\|\cdot\|$ ). Hence, using the standard separation theorem (see Theorem 2.7.8 in [4]), there is a non-zero $\tilde{z}^{*} \in \mathfrak{Z}^{*}$ such that

$$
\tilde{z}^{*}(z) \geq 0 \text { for all } z \in H_{\hat{x}}(\overline{[Q \mid \theta]} \cap O)-[Z \mid h(\hat{x})]^{0} .
$$

By virtue of Lemma 1 and (6), this implies that

$$
\tilde{z}^{*}(z) \leq 0 \leq \tilde{z}^{*}\left(H_{\hat{x}}(x)\right) \text { for all } z \in \overline{[Z \mid h(\hat{x})]} \text { and } x \in \overline{[Q \mid \theta]} \cap O,
$$

because $H_{\hat{x}}(\overline{[Q \mid \theta]} \cap O)$ and $\overline{[Z \mid h(\hat{x})]}$ are both convex cones. Let us define a norm $\|\cdot\|$ on the product space $R \times \mathfrak{Y} \times R$ by $\|(\xi, y, \zeta)\|=|\xi|+\|y\|+|\varsigma|$ and let us define subsets $B_{1}$ and $B_{2}$ of $R \times \mathfrak{Y} \times R$ as follows:

$$
\begin{gathered}
B_{1}=\{(\xi, y, \varsigma) ; \xi<0, y \in \operatorname{int.}[Y \mid g(\hat{x})], \varsigma<0\}, \\
B_{2}=\left\{\left(F_{\hat{x}}(x), G_{\hat{x}}(x), \tilde{z}^{*}\left(H_{\hat{x}}(x)\right)\right) ; x \in \overline{Q Q \mid \theta]} .\right.
\end{gathered}
$$

Since $\varnothing \neq$ int. $Y \subset[Y \mid g(\hat{x})]$ it is easily verified that $B_{1}$ is an open convex cone in $R \times \mathfrak{Y} \times R$. Since $\overline{[Q \mid \theta]}$ is a convex cone in $\mathfrak{X}$ such that $\overline{[Q \mid \theta]} \cap O=\varnothing$, the linearity of the mapping $\left(F_{\hat{x}}(\cdot), G_{\hat{x}}(\cdot), \tilde{z}^{*}\left(H_{\hat{x}}(\cdot)\right)\right)$ implies that $B_{2}$ is a convex cone in $R \times \mathfrak{Y} \times R$. In addition, it follows from (10) that $B_{1} \cap B_{2}=\varnothing$. We may again use the standard separation theorem to claim the existence of $\eta \in R$, $y^{*} \in \mathfrak{Y}^{*}$ and $\tilde{\eta} \in R$ such that

$$
\begin{gathered}
\left(\eta, y^{*}, \tilde{\eta}\right) \neq\left(O, \theta^{*}, O\right), \quad \eta \geq 0, \tilde{\eta} \geq 0, \\
y^{*}(y) \leq 0 \text { for all } y \in \operatorname{int} .[Y \mid g(\hat{x})] \text { and } \\
\eta \cdot F_{\hat{x}}(x)+y^{*}\left(G_{\hat{x}}(x)\right)+\tilde{\eta} \cdot \tilde{z}^{*}\left(H_{\hat{x}}(x)\right) \geq 0 \text { for all } x \in \overline{[Q \mid \theta]} .
\end{gathered}
$$

Since $\hat{x}$ is non-singular, (ii) of the Definition together with (10)-(13) imply that on setting $z^{*}=\tilde{\eta} \cdot \tilde{z}^{*}$ we have

$$
\begin{gathered}
\eta \geq 0, \quad\left(\eta, y^{*}\right) \neq\left(O, \theta^{*}\right), \\
y^{*}(y) \leq 0 \text { for all } y \in \operatorname{cl} .[Y \mid g(\hat{x})], \\
z^{*}(z) \leq 0 \text { for all } z \in \operatorname{cl} .[Z \mid h(\hat{x})] \text { and } \\
\eta \cdot F_{\hat{x}}(x)+y^{*}\left(G_{\hat{x}}(x)\right)+z^{*}\left(H_{\hat{x}}(x)\right) \geq 0 \text { for all } x \in[Q \mid \theta] .
\end{gathered}
$$

We now consider the case where $\overline{[Q \mid \theta]} \cap O \neq \varnothing$. Let us define a norm on the product space $R \times \mathfrak{Y}$ by $\|(\xi, y)\|=|\xi|+\|y\|$. We can easily show that $F_{\hat{x}}(\overline{[Q \mid \theta]}) \times$ $G_{\hat{x}}(\overline{[Q \mid \theta]})$ is a convex cone in $R \times \mathfrak{Y}$. Set $D=\{(\xi, y) ; \xi<0, y \in \operatorname{int} .[Y \mid g(\hat{x})]\}$. The set $D$ is an open convex cone in $R \times \mathfrak{Y}$ such that $D \cap F_{\hat{x}}(\overline{[Q \mid \theta]}) \times G_{\hat{x}}(\overline{[Q \mid \theta]})=$ $\varnothing$. Using the standard separation theorem, there exist $\eta \in R$ and $y^{*} \in \mathfrak{Y}^{*}$, not both zero, such that

$$
\eta \cdot \xi+y^{*}(y) \leq 0 \text { for all } \xi<0, y \in \text { int. }[Y \mid g(\hat{x})]
$$


and

$$
\eta \cdot F_{\hat{x}}(x)+y^{*}\left(G_{\hat{x}}(x)\right) \geq 0 \text { for all } x \in \overline{[Q \mid \theta]} .
$$

If we set $z^{*}=\theta^{*}\left(\in 3^{*}\right)$ it follows from (18) and (19) that (14)-(17) also hold. Therefore we conclude that there exist $\eta \in R, y^{*} \in \mathfrak{Y}^{*}$ and $z^{*} \in \mathfrak{Z}^{*}$ such that (14)-(17) hold, whether or not the set $\overline{[Q \mid \theta]} \cap O$ is empty. Since $\hat{x}$ is a feasible solution of $(\mathrm{P})$ it is easily verified that $\pm g(\hat{x}) \in \mathrm{cl} .[Y \mid g(\hat{x})]$ and $\pm h(\hat{x}) \in$ cl. $[Z \mid h(\hat{x})]$. Hence by virtue of (14)-(17) we conclude that the lemma holds.

PROOF OF THEOREM 1. By virtue of Lemma 3, it suffices to show that (9) holds in order to show our desired results. We will show this by contradiction. Let us assume that there is an $\tilde{x} \in\left[\overline{Q \mid \theta]} \cap O\right.$ such that $H_{\hat{x}}(\tilde{x}) \in[Z \mid h(\hat{x})]^{0}$. It follows, from (1) and (v) of Lemma 2, that there are $\mu_{g}>0$ and $\mu_{h}>0$ such that $F_{\hat{x}}(\tilde{x})<0, G_{\hat{x}}(\tilde{x}) \in \mu_{g}$ (int. $\left.Y-g(\hat{x})\right)$ and $H_{\hat{x}}(\tilde{x}) \in \mu_{h}\left(Z^{0}-h(\hat{x})\right)$. By virtue of (i) of the Definition this implies that there is a $\delta>0$ such that

$$
\begin{gathered}
\{f(\hat{x}+\lambda u)-f(\hat{x})\} / \lambda<0, \\
\{g(\hat{x}+\lambda u)-g(\hat{x})\} / \lambda+\mu_{g} g(\hat{x}) \in \text { int. } Y, \\
\{h(\hat{x}+\lambda u)-h(\hat{x})\} / \lambda+\mu_{h} h(\hat{x}) \in Z^{0},
\end{gathered}
$$

whenever $0<\lambda<\delta$ and $|\tilde{x}-u|<\delta$. We know that $T(X, \hat{x})$ is a cone which is closed with respect to the additional norm $|\cdot|$ (see e.g., [8]). Hence it can be verified that $\tilde{x} \in \overline{[Q \mid \theta]} \subset T(X, \hat{x})$. By virtue of (2) this implies that there are sequences $\left\{\lambda_{n}\right\}$ and $\left\{x_{n}\right\}$ such that

$$
\begin{aligned}
& \lambda_{n}>0, x_{n} \in X \quad \text { for each } n=1,2, \ldots \quad \text { and } \\
& \lim _{n \rightarrow \infty} \lambda_{n}=0, \quad \lim _{n \rightarrow \infty}\left|\tilde{x}-\left(x_{n}-\tilde{x}\right) / \lambda_{n}\right|=0 .
\end{aligned}
$$

Hence there is a positive integer $k$ such that

$$
\begin{gathered}
0<\lambda_{k}<\delta, \quad\left|\tilde{x}-\left(x_{k}-\hat{x}\right) / \lambda_{k}\right|<\delta, \\
0<\mu_{g} \lambda_{k}<1 \quad \text { and } 0<\mu_{h} \lambda_{k}<1 .
\end{gathered}
$$

By virtue of (20)-(22) and (iii) of Lemma 2 and because $g(\hat{x}) \in Y, h(\hat{x}) \in Z$ and $x_{k}=\hat{x}+\lambda_{k}\left[\left(x_{k}-\hat{x}\right) / \lambda_{k}\right]$, we have

$$
\begin{array}{cl}
g\left(x_{k}\right) \in \operatorname{int} . Y, & h\left(x_{k}\right) \in Z^{0}, \quad x_{k} \in X \quad \text { and } \\
& f\left(x_{k}\right)<f(\hat{x}) .
\end{array}
$$

Since $\hat{x}$ is an optimal solution of (P), it follows from (23) that $f\left(x_{k}\right) \geq f(\hat{x})$, which contradicts (24). Hence we conclude that the relation (9) holds, completing the proof of Theorem 1. 


\section{An example and concluding remarks}

In this section, let us consider the following optimisation problem:

$$
\text { minimise } \int_{0}^{1}\left(-\int_{0}^{t} x(s) d s\right) d t
$$

üinàer ine constraints

$$
\begin{aligned}
& x \in \mathfrak{C}, \quad \int_{0}^{1} \sqrt{1+x(t)^{2}} d t \leq \frac{\pi+4}{4 \sqrt{2}} \text { and } \\
& x(t) \leq 1 \quad \text { for all } t \in 1,
\end{aligned}
$$

where $I=[0,1], \pi$ is the circle ratio and $\mathfrak{C}$ is the normed space of all continuous real-valued functions defined on $I$ with the following norm:

$$
\|x\|=\left[\int_{0}^{1}|x(t)|^{2} d t\right]^{1 / 2} \quad \text { for each } x \in \mathfrak{C} .
$$

Let us define convex cones $Y \subset R$ and $Z \subset \mathfrak{C}$ as follows:

$$
Y=\{\xi ; \xi \leq 0\}, \text { and } Z=\{x \in \mathfrak{C} ; x(t) \leq 0 \text { for all } t \in I\} .
$$

Note that $Y$ has non-empty interior. We define the mappings $f: \mathfrak{C} \rightarrow R, g: \mathfrak{C} \rightarrow$ $R$ and $h: \mathfrak{C} \rightarrow \mathfrak{C}$ as follows: for each $x \in \mathfrak{C}$,

$$
\begin{gathered}
f(x)=\int_{0}^{1}\left(-\int_{0}^{t} x(s) d s\right) d t, \\
g(x)=\int_{0}^{1} \sqrt{1+x(t)^{2}} d t-\frac{\pi+4}{4 \sqrt{2}} \text { and } \\
h(x)(t)=x(t)-1 \quad \text { for all } t \in I .
\end{gathered}
$$

Then our problem consisting of (25) and (26) is now rewritten as the problem (P), where $\mathfrak{X}=\mathfrak{Z}=\mathfrak{C}, \mathfrak{Y}=\mathfrak{R}$ and $X=\mathfrak{C}$. Let us define the additional norm on $\mathfrak{C}$ as follows:

$$
|x|=\sup _{t \in I}|x(t)| \quad \text { for each } x \in \mathfrak{C} .
$$

Then one can easily show that the convex cone $Z$ has a non-empty interior with respect to the additional norm $|\cdot|$ and that $\|x\| \leq|x|$ for each $x \in C$. Hence our problem satisfies the Assumption in Section 1.

Using Theorem 1, we seek an optimal solution of our problem. On obtaining this solution we shall verify its non-singularity. Let $\hat{x} \in \mathbb{C}$ be an optimal solution of our problem. We set $Q=\mathfrak{C}$ and we define the linear mappings $F_{\hat{x}}: \mathfrak{C} \rightarrow R$, $G_{\hat{x}}: \mathfrak{C} \rightarrow R$ and $H_{\hat{x}}: \mathfrak{C} \rightarrow \mathfrak{C}$ as follows: for each $x \in \mathfrak{C}$,

$$
F_{\hat{x}}(x)=\int_{0}^{1}\left(-\int_{0}^{t} x(s) d s\right) d t
$$




$$
\begin{gathered}
G_{\hat{x}}(x)=\int_{0}^{1} \frac{\hat{x}(t)}{\sqrt{1+\hat{x}(t)^{2}}} x(t) d t \quad \text { and } \\
H_{\hat{x}}(x)(t)=x(t) \quad \text { for all } t \in I .
\end{gathered}
$$

We know that, for any $z^{*} \in \mathfrak{C}^{*}$, there is a Lebesgue integrable function $\psi(t)$ defined on $I$ such that

$$
z^{*}(z)=\int_{0}^{1} \psi(t) z(t) d t \quad \text { for all } z \in \mathfrak{C}
$$

where the integral is to be interpreted in the sense of Lebesgue. By paying attention to this and noting as well that $Q=\mathfrak{C}$, it follows, by virtue of Theorem 1 , that there are non-negative numbers $\eta, \mu$, not both zero, and a square Lebesgueintegrable function $\psi(t)$ defined on $I$, such that

$$
\begin{gathered}
\mu\left(\int_{0}^{1} \sqrt{1+\hat{x}(t)^{2}} d t-\frac{\pi+4}{4 \sqrt{2}}\right)=0 \\
\psi(t) \geq 0, \quad \psi(t)(\hat{x}(t)-1)=0 \quad \text { for almost all } t \in I, \\
\eta(t-1)+\frac{\mu \hat{x}(t)}{\sqrt{1+\hat{x}(t)^{2}}}+\psi(t)=0 \quad \text { for almost all } t \in I .
\end{gathered}
$$

One can obtain the following form of (27)-(29) with a little effort.

$$
\begin{gathered}
\hat{x}(t)= \begin{cases}1 & \text { for } 0 \leq t \leq \frac{1}{2} \\
\frac{\sqrt{2}(1-t)}{\sqrt{1-2(1-t)^{2}}} & \text { for } \frac{1}{2}<t \leq 1,\end{cases} \\
n=1, \quad \mu=\frac{1}{\sqrt{2}} \text { and } \\
\psi(t)= \begin{cases}\frac{1}{2}-t & \text { for } 0 \leq t \leq \frac{1}{2} \\
0 & \text { for } \frac{1}{2}<t \leq 1 .\end{cases}
\end{gathered}
$$

Now we shall verify that $\hat{x}$ given by (30) is a non-singular point of our problem. We can easily verify that the mappings $F_{\hat{x}}, G_{\hat{x}}$ and $H_{\hat{x}}$ satisfy (i) of the Definition. Because $Q=T(\mathfrak{C}, \hat{x})=\mathfrak{C}, Q$ is a convex subset of $\mathfrak{C}$ with $\theta \in Q \subset T(Q, \hat{x})$. That is, (ii) of the Definition is satisfied also. Let $z \in \mathfrak{C}$ be arbitrary. We set $\delta=\|z\|$. We shall consider the case where $\delta>0$. We set $z_{-}(t)=[|z(t)|-z(t)] / 2$ for all $t \in I$ and

$$
\varepsilon=\min .\left\{\frac{1}{4}, \frac{3 \delta^{2}}{\left[z_{-}(1 / 2)+\delta\right]^{2}}\right\} \quad(>0) .
$$

Furthermore let us define a continuous function $x(t)$ on $I$ as follows:

$$
x(t)= \begin{cases}-z_{-}(t)-\delta & \text { for } 0 \leq t \leq \frac{1}{2} \\ \left\{\left(z_{-}(1 / 2)+\delta\right)(t-\varepsilon-1 / 2)\right\} / \varepsilon & \text { for } \frac{1}{2}<t \leq \frac{1}{2}+\varepsilon \\ 0 & \text { for } \frac{1}{2}+\varepsilon<t \leq 1\end{cases}
$$


Then it can be verified that

$$
x \in Q=\overline{[Q \mid \theta]}(=\mathfrak{C})
$$

and that there is an $\tilde{\varepsilon} \in(0, \varepsilon)$ such that

$$
x(t)<-z_{-}(t) \text { whenever } \frac{1}{2} \leq t \leq \frac{1}{2}+\tilde{\varepsilon} .
$$

This foliows from the fact that $x(t)$ and $z_{-}(t)$ are both continuous functions on $I$ such that $x(1 / 2)<-z_{-}(1 / 2)$. We set

$$
\begin{gathered}
\lambda=\frac{\sup _{t \in I}\left|z_{-}(t)\right|+\delta}{1-\hat{x}(1 / 2+\tilde{\varepsilon})} \quad(>0), \\
\tilde{z}(t)=\frac{x(t)-z(t)}{\lambda}+\hat{x}(t)-1 \quad \text { for all } t \in I .
\end{gathered}
$$

Since $1-\hat{x}(1 / 2+\tilde{\varepsilon})>0$ and $\hat{x}(t)$ is a decreasing function on the closed interval $[1 / 2,1]$, it can be verified that $\tilde{z} \in Z$ and $z=H_{\hat{x}}(x)-\lambda[\tilde{z}-h(\hat{x})]$. Hence we obtain that

$$
z \in H_{\hat{x}}(x)-\overline{[Z \mid h(\hat{x})]} \text {. }
$$

Furthermore, by the definition of $x$, we can verify that

$$
\|x\| \leq \sqrt{5}\|z\| \text {. }
$$

If $\delta=0$, we let $x=\theta(\in \mathfrak{C})$. It is then evident that (31)-(33) hold, because $\theta \in \overline{[Z \mid h(\hat{x})]}$. Hence we can now conclude that the optimal solution given by (30) is a non-singular point of our problem, by setting $K=\mathfrak{C}(=3)$. Moreover the optimal value can be calculated to be:

$$
\int_{0}^{1}\left(-\int_{0}^{t} \hat{x}(x) d s\right) d t=-\frac{\pi+4}{16}
$$

Let us now state a concluding remark. We can apply Theorem 3.2 in [7] to our example, because $Q$ is a convex subset of $\mathfrak{C}$ with $\theta \in Q \subset T(\mathfrak{C}, \hat{x})$ and the space $\mathfrak{C}$ is complete with respect to the additional norm $|\cdot|$. In this case the linear functional $z^{*}$ in Theorem 1, however, is continuous with respect to the additional norm $|\cdot|$ but it is not always continuous with respect to the original norm $\|\cdot\|$. Hence (28) and (29) would be respectively rewritten as follows:

$$
\begin{gathered}
\psi(t) \text { is non-decreasing function defined on } I, \\
\int_{0}^{1}(\hat{x}-1) d \psi(t)=0, \\
\int_{0}^{1}\left\{n(t-1)+\frac{\mu \hat{x}(t)}{\sqrt{1+\hat{x}(t)^{2}}}\right\} x(t) d t+\int_{0}^{1} x(t) d \psi(t) \geq 0 \\
\quad \text { for all } x \in \mathfrak{C},
\end{gathered}
$$


where the integrals in (35) and the latter half of (36) are in the sense of Stieltjes. We must recognise that there is a great difference between the relations (28), (29) and those of (34)-(36). We now end our discussion by emphasising again that it is important to give an assurance that the linear functional $z^{*}$ in Theorem 1 is continuous with respect to the original norm $\|\cdot\|$.

\section{Acknowledgement}

The author is greatly indebted to the referees for detailed corrections and valuable suggestions, and wishes to thank Prof. Kaneda of Yamaguchi University for his valuable comments.

\section{References}

[1] P. J. Bender, "Nonlinear programming in normed linear spaces", J. Optim. Theory Appl. 24 (1978) 263-285.

[2] J. Borwein, "Weak tangent cones and optimization in a Banach space", SIAM J. Control Optim. 16 (1978) 512-522.

[3] B. D. Craven, "Lagrangean conditions and quasiduality", Bull. Austral. Math. Soc. 16 (1977) 325-339.

[4] N. Dunford and J. T. Schwartz, Linear operators Part I: General theory (Interscience, New York, 1964).

[5] R. B. Holmes, Geometric functional analysis and its applications (Springer-Verlag, New York, 1975).

[6] S. Kurcyusz, "On the existence and nonexistence of Lagrange multipliers in Banach spaces", J. Optim. Theory Appl. 20 (1976) 81-110.

[7] Y. Nagahisa and Y. Sakawa, "Nonlinear programming in Banach spaces", J. Optim. Theory Appl. 4 (1969) 182-190.

[8] P. P. Varaiya, "Nonlinear programming in Banach space", SIAM J. Appl. Math. 15 (1957) 284-293.

[9] J. Zowe and S. Kurcyusz, "Regularity and stability for the mathematical programming in Banach spaces", Appl. Math. Opt. 5 (1979) 49-64. 